\title{
Acerca del carácter representacional de la mente. La mente representacional
}

\author{
José María Zumalabe Makirriain \\ Universidad del País Vasco, España
}

(Recibido 20 Octubre, 2011; Aceptado 8 Julio, 2013)

RESUMEN: Desde las ciencias cognitivas se entiende el pensamiento en términos de estructuras de representaciones mentales sobre las que operan procesos computacionales. En el modelo representacional-computacional de la mente se recurre a una compleja analogía triádica que vincula mente, cerebro y ordenadores. La mayoría de estos modelos son simbólicos, aunque también existen modelos representacionales no simbólicos (conexionismo) y modelos cognitivos no representacionales de la mente. El análisis de los diferentes enfoques cognitivos sobre las representaciones y los procesos mentales en el marco de la ciencia cognitiva y de sus ventajas y limitaciones revela que se trata de enfoques que no tienen por qué ser excluyentes entre sí y que en muchos de los casos se complementan, aunque también se constata la ausencia de una teoría unificada al respecto. Tras considerar los puntos débiles tanto del modelo simbólico computacional clásico como del conexionismo, reconociendo los avances significativos propiciados por ambos en el estudio de la mente, se concluye que no existe todavía ningún modelo computacional con capacidad representacional para abarcar todo el pensamiento humano.

Palabras clave: representación mental, procesos computacionales, modelo simbólico clásico, conexionismo, enfoques representacionales.

\section{About the representational nature of the mind. The representational mind.}

\begin{abstract}
According to cognitive science, thinking is understood as structures of mental representations on which computational processes operate. In the representational-computational model of the mind, we resort to a complex triadic analogy that links mind, brain and computers. Most of these models are symbolic, although there are also non-symbolic representational models (connectionism) and non-representational cognitive models of the mind. The analysis of the various cognitive approaches on the representations and the mind processes within the framework of the cognitive sciences and of their advantages and limitations reveals that these approaches do not have to mutually exclusive and that, in many of the cases, they complement each other. However, the lack of a unified theory regarding this matter has also been stated. After considering the weak points of both the classic computational symbolic model and the connectionism, acknowledging the significant progresses made by both in the study of the mind, we conclude that there is still no computational model with representational capacity to cover the whole human thinking.
\end{abstract}


Keywords: mental representation, computational processes, classic symbolic model, connectionism, representational approaches.

Desde las ciencias cognitivas se considera que el pensamiento es el producto de representaciones mentales y procesos computacionales que operan sobre esas representaciones. La información en una representación interna puede referirse a todo aquello que podemos conocer del mundo. Cuando pensamos, manipulamos las representaciones mentales de objetos, actividades y situaciones. Una representación es una estructura que simboliza algo en virtud de relaciones de semejanza, causalidad o de vínculos con otras representaciones. Se trata de una estructura o una actividad simbólica que se construye para codificar la experiencia. "La concreción de las representaciones mentales es la vía que conduce al rigor en psicología“" (Pinker, 2007, p. 121).

En ciencia cognitiva coexisten planteamientos teóricos y metodológicos que mantienen el carácter representacional de la mente (modelo cognitivo simbólico clásico y modelo conexionista), y planteamientos no representacionales. En el siguiente esquema se describen someramente los puntos de vista más destacados dentro de esta diversidad:

\section{I-modelos representacionales de la mente}

- Se entiende el pensamiento en términos de estructuras de representaciones mentales sobre las que operan procesos computacionales. La cognición es un tipo de computación (Pylyshyn, 1984).

- Los procesos de información que maneja un sistema computacional tienen un carácter representacional. Todo sujeto debe contar con algún sistema de representaciones mentales que contiene información sobre el mundo y sobre sí mismo.

- Existe una relación causal entre la representación cognitiva del sujeto y su conducta.

\section{A) Modelos simbólicos}

Modelo simbólico clásico (Fodor, 1985, 1988; Newell y Simon, 1972; Pylyshyn, 1984, 2002).

- La representación en la mente se realiza mediante símbolos discretos y significativos que se combinan mediante reglas sintácticas para formar expresiones significativas.

- Un sistema de representaciones simbólico constituye el "lenguaje de la mente" que tiene un vocabulario, una estructura sintáctica y una semántica.

- Las representaciones mentales son sistemas de de símbolos físicos; en los humanos sistemas de procesos u objetos neuronales. Pensar sería una función cerebral por lo que la información debe estar representada en el cerebro 
de alguna manera.

- La representación cognitiva se describe en función de símbolos, esquemas imágenes ideas y otras formas de representación mental que sirven de base para que el sujeto actúe.

- Plantean un único procesador central que ejecuta cómputos simbólicos secuencialmente; se trata de un procesamiento en serie en el que el pensamiento avanza paso a paso.

\section{B) Modelos no simbólicos}

Modelos conexionistas (Rumelhart, 1980, 1989; Rumelhart, McClelland and PDP, 1986)

- Proponen un modelo de pensamiento formado por redes neuronales artificiales, recurriendo a una compleja analogía triádica que vincula la mente, el cerebro y los ordenadores.

- La cognición se produce a partir de la interacción simultánea de una serie de unidades semejantes a neuronas que se encuentran altamente interconectadas. Se utilizan las neuronas y sus conexiones como modelo de estructuras de datos y el estímulo de las células nerviosas y la diseminación de la actividad neuronal como modelo de algoritmos.

- La naturaleza del sistema es neurofisiológica ya que las representaciones están neurológicamente distribuidas y sus estados de actividad corresponden a patrones de actividad neural.

- Al ser modelos no simbólicos, los pesos de las conexiones determinan el funcionamiento de la red. La actividad mental surge de la fuerza de las conexiones entre las unidades del sistema, no de los símbolos codificados en él, determinando éstas conexiones el funcionamiento de la red.

- Desarrollan modelos compuestos por un gran número de procesadores o nodos interconectados que funcionan en paralelo, procesamiento en el que se dan muchos pasos simultáneamente.

- Para estudiar la mente se refieren a parámetros como: patrones de conectividad, unidades subsimbólicas definidas por niveles de activación, fuerzas de conexión o los umbrales de de input y output de tales unidades.

- No se precisa de un programa almacenado ni de un lenguaje interior constituido por la manipulación de símbolos. Al no existir un programa almacenado en las redes, las representaciones se manipulan de manera no computacional partiendo de las representaciones que constituyen el input del sistema y de otro conjunto de representaciones que constituyen su output, sin representaciones intermedias.

- La representación es numérica en vez de simbólica y las unidades de procesamiento se comunican entre ellas a través de señales numéricas en lugar de señales simbólicas.

- Las neuronas individuales no representan símbolos; es la pauta de actividad de grandes agrupaciones neuronales distribuidas en el cerebro lo que corres- 
ponde a una representación.

\section{II-modelos no representacionales}

Cognición incorporada (Clark, 1998).

Realismo incorporado (Johnson y Lakoff. 2002).

Enfoques dinámicos y emergentistas.

En general una representación tiene dos funciones básicas:

a) Está dirigida a, es decir que significa, señala, indica, se refiere a o representa un objeto o situación, y b) Dice algo acerca de ese objeto o estado de cosas. La primera de las funciones haría referencia al objeto de la representación y la segunda al contenido de la misma.

Los procesos de información pueden ser descritos formalmente, lo que implica una relación con la noción de algoritmo, que es definida en términos de procesos que operan sobre la representación. "Un algoritmo es un procedimiento o sistema formal, que es definido más en términos de la forma de la representación que de su significado. Es un asunto puramente de manipulación de patrones en la representación“ (Medina, 2008, p. 187). La psicología cognitiva, desde sus inicios se caracterizó por la tendencia de ofrecer explicaciones formales de los fenómenos, definiendo su génesis en virtud de la forma de los algoritmos que los producen.

El análisis a nivel de algoritmos, se refiere a cómo se representan las entradas y salidas del sistema, y a la forma en que la entrada se transforma en producción. Una aproximación a la descripción de un procesamiento de la información en el nivel algorítmico, consiste en identificar el problema general, y luego descomponer éste en subobjetivos que, a su vez, pueden descomponerse en otros subobjetivos. Se trata del proceso al que Cummins (1983) denomina análisis funcional.

El organismo actúa como un sistema que realiza una serie de operaciones formales sobre la información traducida en códigos cognitivos, que tienen una relación representacional directa de un dominio particular.

Las representaciones dependen de los procesos y estos son los que determinan la relación existente entre los elementos del objeto representado. Con frecuencia, la representación hace referencia a una estructura sobre la que se ejecutan determinados procesos. Estos procesos se refieren a las actividades implicadas en la utilización de la información almacenada en una determinada estructura o representación. Aunque con relativa frecuencia no resulta sencillo distinguir entre estructura representacional y procesos psicológicos (Paivio, 1986).

Uno de los problemas centrales que se plantea en psicología cognitiva se refiere a la explicación de cómo se representa el conocimiento (Amsel, 1989; Holland, Holyoak, Nisbett y Thagard, 1986; Marslen-Wilson, 1989; Paivio, 1986; 
Palmer y Kimchi, 1986; Smythe, 1988, 1989). Son muchas las teorías elaboradas para explicar cómo se codifica, almacena y recupera el conocimiento que han dado lugar a una variedad de formalismos representacionales diferentes como: redes semánticas (Collins y Loftus, 1975), marcos (Minsky, 1975), sistemas de producción (Newell, 1973; Anderson, 1983), imágenes mentales (Kosslyn, 1980; Paivio, 1986; Shepard y Cooper, 1982), representaciones estructurales (Schacter, Cooper y Delaney, 1990), etc. En todos ellos se recurre a una compleja analogía triádica que vincula la mente, el cerebro y los ordenadores.

De la misma manera que en el paradigma simbólico-computacional se seguía la metáfora del ordenador, los modelos conexionistas están mucho más próximos a la metáfora del cerebro. Los conexionistas definen la mente como un sistema de cómputo que se encarna en un tipo especial de procesador de información: el sistema nervioso. Lo que se pretende desde el conexionismo es desarrollar modelos de cómputos que simultáneamente sean respetuosos con los datos de conducta y con los aspectos computacionalmente relevantes del funcionamiento del sistema nervioso. Y este objetivo se establece con la finalidad de resolver las limitaciones detectadas en el paradigma simbólico-computacional clásico.

Desde el conexionismo se intenta ofrecer descripciones cognitivas construidas a partir de entidades que corresponden a los elementos constituyentes de los símbolos utilizados en el paradigma simbólico, es decir que trata con unidades a nivel subsimbólico. Este nivel tiene su propia semántica que es diferente de la del nivel simbólico o conceptual. En el paradigma simbólico las entidades se representan mediante símbolos, sin embargo, en el paradigma subsimbólico se representan mediante un gran número de subsímbolos que participan en cómputos numéricos, no simbólicos (Smolensky, 1988).

Ambos modelos cognitivos asignan contenido semántico: el modelo clásico a las expresiones semánticas y el PDP (Paralled Distributed Processing) a los nodos; los modelos conexionistas reconocen exclusivamente conexiones causales entre nodos y los modelos simbólicos lo hacen entre objetos evaluables semánticamente. En el conexionismo, el "input" del sistema surge de los valores numéricos de activación de las unidades "input" de la red, valores que suponen una representación del "input. Esta representación “...se propaga a lo largo de las conexiones hasta que aparece algún conjunto de valores de activación en las unidades del "output". Los valores de activación codifican el "output" que el sistema ha computado a partir del "input" (Ballesteros, 1992, p. 348).

\section{Enfoques sobre las representaciones.}

Son muchos los tipos de representaciones y operaciones computacionales que son esenciales para comprender el pensamiento humano, que no tienen por que ser excluyentes entre sí y que en muchos de los casos se complementan. A 
continuación, nos vamos a referir someramente a algunos de los enfoques que consideramos más relevantes.

La lógica se dedica al estudio del razonamiento válido y aunque no ha sido uno de los enfoques más influyentes en el estudio psicológico de la representación mental muchas de las ideas fundamentales sobre la representación y la computación surgieron en este ámbito.

En la década de 1930 fueron desarrolladas las primeras teorías computacionales por lógicos como Church y Turing entre otros, que idearon esquemas matemáticos para determinar qué problemas podían resolverse por medio de procesos computacionales.

En lógica, las conclusiones se extraen por medio de la aplicación de reglas de inferencia a un conjunto de premisas y todavía hoy son muchos los filósofos e investigadores del campo de la inteligencia artificial que se valen de la lógica para estudiar el razonamiento. Algunos lingüistas ven la lógica formal como una herramienta natural para comprender la estructura del lenguaje (McCawley, 1993); otros como Stabler (1992) recurren a la lógica para formalizar algunas de las ideas más recientes de Noam Chomsky.

Las reglas son estructuras de tipo condicional: si A, entonces $\mathrm{B}$ que tienen una serie de propiedades representacionales y computacionales. Los modelos de reglas desde un principio han sido aplicados a la psicología, al contrario que la mayoría de los modelos computacionales lógicos que no fueron concebidos como modelos de la cognición humana.

El primer programa de inteligencia artificial fue el Teórico Lógico (Newell, Shaw y Simon, 1958) que, introducido en un ordenador, resolvía ejercicios de lógica formal comportándose como un modelo de razonamiento lógico humano que incluía reglas de inferencia y reglas estratégicas para la búsqueda eficiente de pruebas. Este modelo derivó en el Solucionador General de Problemas (General Problem Solver o GPS; Newell y Simon, 1972) que se puede considerar el primer marco general para el estudio del pensamiento humano que utilizaba reglas para simular soluciones humanas para diferentes tipos de problemas. Posteriormente aparecieron dos sistemas cognitivos de reglas de gran influencia en las ciencias cognitivas por su gran aplicabilidad a la cognición humana: el ACT (Adaptative Control of Thought; Anderson, 1983, 1993) y el SOAR (State, Operador, And Result; Newell, 1990; Rosenbloom, Laird y Newell, 1993), ambos con enormes posibilidades de aplicación en psicología y computación.

El procesamiento que se basa en las reglas puede ser tanto serial (una regla cada vez) como paralelo (varias reglas en un mismo paso), pero habitualmente el pensamiento consciente suele producirse en serie, es decir haciendo una in- 
ferencia tras otra. Muchas formas de aprendizaje se representan fácilmente en términos de la adquisición, la modificación y la aplicación de reglas. Algunas reglas pueden formar parte de los mecanismos biológicos de los que estamos dotados los humanos cuando nacemos, es decir, que pueden ser innatas aunque la mayoría de ellas son aprendidas, o pueden inferirse de otras reglas.

Chomsky (1972) sostenía la idea de que los seres humanos nacemos con una gramática universal innata, aunque más tarde planteaba la idea de que la adquisición del lenguaje consiste en el reconocimiento automático de un conjunto finito de parámetros y de su configuración particular para una lengua determinada (Chomsky, 1988). Más recientemente Chomsky (2002) llega a poner en tela de juicio su concepción de la gramática como un sistema de reglas.

Los sistemas de reglas constituyen el enfoque computacional-representacional que ha tenido la mayor cantidad de aplicaciones psicológicas. Newell (1990) ha mostrado las aplicaciones del modelo SOAR en una enorme cantidad de fenómenos psicológicos. Holland et al. (1986) utilizan sistemas de reglas para explicar muchos tipos diferentes de aprendizaje y Anderson $(1983,1993)$ ha utilizado sistemas de reglas (ACT) para explicar la adquisición y el uso del lenguaje, así como para dar cuenta de la adquisición de capacidades como la resolución de problemas geométricos o la programación informática.

Además, Anderson, Qin, Stenger y Carter (2004) han relacionado con áreas específicas del cerebro la nueva versión del sistema ACT denominada ACT-R, concluyendo que las reglas se aplican en los ganglios basales (núcleos ubicados en la materia blanca de la corteza cerebral) y que los hechos que se corresponden con las reglas se almacenan en la corteza prefrontal, extendiendo al ámbito neurológico un modelo que se originó como puramente cognitivo.

Los modelos computacionales basados en reglas han originado simulaciones muy detalladas de una enorme cantidad de procesos psicológicos y han mostrado su utilidad práctica en diferentes campos de aplicación (enseñanza, lenguaje, inteligencia artificial, aprendizaje,...).

Los conceptos son representaciones mentales de una clase de objetos o hechos relacionados entre sí que normalmente se corresponden con una palabra. Se trata de una clase de representación mental que agrupa objetos, relaciones, actividades, abstracciones o características que tienen propiedades comunes. Aunque algunos autores como el filósofo de la mente Fodor (1985) defiendan la hipótesis del innatismo, en la actualidad las ciencias cognitivas estudian los procesos que intervienen en la formación de conceptos a partir de la experiencia y de otros conceptos.

En la década de 1970 se hizo más patente el interés de los psicólogos por 
el estudio de la naturaleza de los conceptos y se crearon términos como marco, esquema y guión, que son formas más complejas que los conceptos de correspondencia con las palabras, pero son similares a los conceptos en el sentido de que también están constituidos por conjuntos de rasgos con los que pueden encontrarse correspondencias para aplicarlos a situaciones nuevas.

Entendidos como marcos, esquemas o guiones, los conceptos son representaciones de individuos o situaciones típicas y no definiciones y por consiguiente expresan características y no verdades universales. En este sentido, los conceptos constituyen una forma fundamental de organización del conocimiento que no puede expresarse en sistemas de reglas. Esta organización de los conceptos se establece en estructuras jerárquicas (entre las reglas no hay jerarquías) que les confieren propiedades computacionales que los conjuntos de reglas no poseen (Thagard, 2008). Hoy se reconoce que los conceptos no pueden constituir una teoría completa de la representación mental, aunque también se asume que las propiedades computacionales de las representaciones conceptuales hacen de los conceptos un complemento fundamental de las reglas en la modelización del pensamiento humano.

La teoría de prototipos guarda mucha similitud con el enfoque computacional de los conceptos; ahora bien, existe evidencia empírica (Barsalou, 1983; Barsalou, Simmons, Barbey y Wilson, 2003) de que los conceptos son mucho más flexibles y dependientes del contexto que los conjuntos de propiedades típicas, por lo que se puede afirmar que la estructura de los conceptos no se agota en los prototipos. Bastantes autores, como Murphy y Medin, (1985) o Keil (1989), han propuesto modelos conceptuales que han superado la teoría de los prototipos.

Miller (1991) analizó la estructura del léxico mental, la formación de palabras y la adquisición del léxico, considerando que el uso del lenguaje depende de la capacidad de almacenar y utilizar los conceptos denotados por los sustantivos, adjetivos y verbos. Langacker (1987) y Lakoff (1987), por su parte, sostienen que las estructuras sintácticas están ligadas a la naturaleza y al significado de los conceptos.

Kosslyn y Koenig (1992) han estudiado la estructura neuronal del léxico mental analizando los déficits de las personas con daño cerebral y Ashby y Waldron (2000) han analizado datos empíricos según los cuales la corteza prefrontal y los ganglios basales están comprometidos en la adquisición de conceptos. En general se puede afirmar que existe suficiente evidencia psicológica, Murphy, (2002) recoge buena parte de ella, de que los conceptos forman parte del conocimiento general del mundo.

La analogía es un proceso mental por el que se establecen relaciones de semejanza entre dos grupos de objetos. El pensamiento analógico consiste en tratar 
situaciones nuevas mediante adaptaciones de situaciones similares ya conocidas. Se trata de un tipo de conocimiento que se basa en la rememoración de las experiencias previas que se parecen a la nueva situación.

Evans (1968) creó el primer modelo computacional del razonamiento analógico y desde entonces son muchos los modelos relacionados con el uso de analogías que se han desarrollado. Así por ejemplo, Holyoak y Thagard (1995) elaboraron una teoría computacional del uso de analogías en humanos y desde la inteligencia artificial se han creado aplicaciones muy interesantes del razonamiento analógico o basado en casos (Kolodner, 1993; Leake, 1996).

En el razonamiento analógico se consideran dos situaciones: el análogo objetivo, que representa a la situación nueva sobre la que se razona, y el análogo fuente, que representa la situación anterior que puede adaptarse y aplicarse al análogo objetivo. La analogía es una relación sistemática entre ellos; ahora bien, para establecer analogías interesantes entre situaciones los análogos deben tener relaciones y rasgos similares.

Los análogos son parecidos a los conceptos y distintos de las reglas y los enunciados lógicos en que agrupan paquetes de información; sin embargo son parecidos a los enunciados simples y distintos a los conceptos en que la información que incluyen describe únicamente una situación en particular. Los esquemas analógicos incluyen información general a diferencia de las representaciones de los análogos fuente y objetivo y a semejanza de las reglas y los conceptos. Cuando se usa un análogo fuente para resolver un problema objetivo, se pueden hacer abstracciones construyendo un esquema analógico que capta lo común a ambos análogos. Los esquemas analógicos son parecidos a los conceptos, aunque no poseen el mismo grado de generalidad ya que hacen generalizaciones partiendo sólo de dos instancias. Además, en muchos casos las emociones juegan un importante papel en la representación de análogos.

Para resolver problemas relacionados con un ámbito en el que se posee mucha experiencia se recurre al conocimiento general que proporcionan las reglas y los conceptos; sin embargo para los casos en que se posee experiencia previa en cierto campo pero el conocimiento general es insuficiente se recurre al razonamiento analógico. En consecuencia, las analogías pueden ser de gran utilidad para las situaciones en las que no se dispone ni del conocimiento conceptual ni del conocimiento basado en reglas.

Las explicaciones analógicas son muy abundantes en las ciencias cognitivas y desempeñan un importante papel en la producción y comprensión del lenguaje ya que constituyen el fundamento del uso de las metáforas, que tienen como mecanismo cognitivo subyacente la comparación sistemática de elementos típica del razonamiento analógico. Se han utilizado profusamente en la resolución de 
problemas, en el aprendizaje y en el uso del lenguaje; sus aportaciones son especialmente valiosas cuando se trata de resolver problemas de explicación, por ello tienen un valor potencial enorme en el campo educativo ya que es bastante común que los profesores recurran a comparaciones entre lo nuevo y lo conocido para facilitar a los estudiantes el entendimiento de lo nuevo.

En lo que a la investigación neurológica se refiere, Boroojerdi et al. (2001) descubrieron que la corteza prefrontal participa en el razonamiento analógico en coherencia con los hallazgos que plantean que el razonamiento en el que se necesita recurrir a relaciones complejas, requiere la participación de la corteza prefontal izquierda (Christoff et al., 2001; Kroger et al., 2002). Otros especialistas han creado modelos computacionales para la analogía utilizando redes neuronales artificiales cuyo comportamiento se aproxima al de las redes neuronales biológicas (Hummel y Hoyoak, 1997, 2003; Eliasmith y Thagard, 2001).

Las imágenes mentales son representaciones que reflejan o se parecen a aquello que representan y son una parte importante del pensamiento y de la construcción de los esquemas cognitivos. Aunque los defensores de la ortodoxia del paradigma simbólico-computacional han sido muy críticos con la noción de imagen mental, los datos experimentales sugieren que algunas representaciones son imágenes.

Paivio (1971), y Shepard y Metzler (1971) fueron los primeros investigadores en llevar a cabo experimentos con imágenes visuales y Kosslyn y Schwartz (1977) y Funt (1980) crearon los primeros modelos computacionales. Aunque existen numerosas evidencias computacionales, psicológicas y neurológicas para afirmar que para pensar la mente utiliza tanto las palabras como las imágenes, algunos especialistas en ciencias cognitivas no creen que las representaciones gráficas separadas de las verbales formen parte del pensamiento (Pylyshyn, 1984, 2002).

La mayor parte de los investigadores que se dedican al estudio de las imágenes mentales han focalizado sus esfuerzos en trabajar sobre las representaciones visuales, aunque no hay ninguna razón, en principio, para excluir las imágenes vinculadas con la percepción no visual. Las imágenes mentales se producen en muchos o quizá en todos los tipos de modalidades sensoriales.

Lakoff y Johnson (1986) sugieren que muchas metáforas se originan en imágenes visuales; Lakoff (1994) señala que buena parte del conocimiento depende de esquemas gráficos, conceptos generales que poseen un componente visual, y desde el enfoque de la gramática cognitiva Langacker (1987) sostiene que las metáforas y las imágenes son fundamentales para las actividades mentales, incluido el procesamiento del lenguaje, pues las imágenes sensoriales ejercen un papel primordial en la estructura conceptual. Estos posicionamientos dejan 
abierta la posibilidad de plantear que el lenguaje no depende exclusivamente de las palabras sino también, en alguna medida, de las imágenes visuales o de otro tipo.

También existen evidencias neurológicas que constatan que las imágenes desempeñan una función en el pensamiento. Kosslyn (1994) ha probado que hay zonas del cerebro involucradas en la percepción visual que también participan en la formación de imágenes mentales. Por una parte, comprobó que los pacientes con daño cerebral (en el lóbulo occipital) que afecta a la capacidad de percepción, tenían afectada la capacidad de formar imágenes visuales; por otra parte, descubrió mediante mediciones de la actividad cerebral que cuando las personas utilizan imágenes mentales para realizar determinadas tareas, se activan las áreas cerebrales que participan en la percepción visual. Kosslyn, Ganis y Thompson (2001) recogen reseñas de los diferentes estudios neurológicos de imágenes visuales, auditivas y motrices.

La utilización de imágenes se ha mostrado útil en el aprendizaje, la educación, la resolución de problemas y el diseño de estrategias para mejorar la memoria (Dehaene, Spelke, Pinel, Stanescu y Tsivkin, 1999; Larkin y Simon, 1987). Aún así, el recurso a los modelos computacionales de las imágenes mentales no es muy frecuente y esto posiblemente sea debido, entre otras razones, a que las herramientas de programación disponibles se adaptan mucho mejor a las representaciones verbales que a las visuales.

Otro enfoque de las representaciones se basa en las conexiones, en el que se propone un modelo de pensamiento formado por redes neuronales artificiales desde el que se considera que los individuos tienen representaciones que operan con unidades de procesamiento simples relacionadas mediante conexiones excitatorias e inhibitorias. Una red neuronal hace referencia a un grupo de neuronas interconectadas y existen procesos que propagan la activación entre unidades a través de sus conexiones, y procesos que modifican esas conexiones. La propagación de la activación y el aprendizaje de las unidades generan la conducta.

Una unidad con una conexión excitatoria con una unidad activa recibirá activación de esta última y una unidad con conexión inhibitoria con una unidad activa, verá disminuida su propia actividad. El resultado dependerá de las interconexiones entre las unidades ya que unas se activan mientras otras se desactivan. Las redes conexionistas están formadas por nodos y conexiones simples que sirven para conocer los procesos psicológicos en los que se requiere la satisfacción de restricciones en paralelo.

Los primeros modelos conexionistas del lenguaje tomaban en consideración la percepción visual y auditiva. McClelland y Rumelhart (1981) sugirieron que el reconocimiento de palabras puede considerarse como un problema de satisfac- 
ción de restricciones en paralelo; McClelland y Elman (1986) desarrollaron un modelo similar para explicar la percepción del habla y Kintsch $(1988,1998)$ creó un modelo conexionista para la comprensión discursiva. Rumelhart y McClelland (1986), desarrollaron un modelo de procesamiento distribuido en paralelo para analizar el aprendizaje de verbos en los niños, sin reglas explícitas, rechazado por Pinker y Prince (1988). McWhinney y Leinbach (1991) en respuesta a las críticas, presentaron un nuevo modelo conexionista que corregía los defectos del anterior, ante lo que Pinker y Ullman (2002) respondieron formulando un modelo no conexionista para explicar el procesamiento del lenguaje y McClelland y Patterson (2002) replicaron defendiendo el enfoque conexionista.

Se han utilizado modelos conexionistas locales de comparación y recuperación analógica para simular los resultados de experimentos psicológicos (Holyoak y Thagard, 1995; Kunda y Thagard, 1996; Spellman y Holyoak, 1993; Wharton et al., 1994). En muchos de ellos se han utilizado técnicas de retropropagación; se trata de un algoritmo de aprendizaje en redes con conexiones hacia adelante que ajusta la fuerza de las conexiones entre neuronas. Seidenberg y McClelland (1989) las utilizaron para construir un modelo de reconocimiento visual de palabras; St. John (1992) para generar distribuciones distribuidas que simulan distintos aspectos de la comprensión discursiva y Bates y Elman (2002) para explicar diferentes aspectos del desarrollo evolutivo, como las razones por las que los niños aprenden más rápidamente unas cosas que otras.

Se ha observado que en el cerebro se produce el aprendizaje de Hebb y otras clases de aprendizaje que recurren al ajuste sináptico (Churchland y Sejnows$\mathrm{ki}, 1992)$. En este tipo de aprendizaje se incrementa la fuerza de las uniones sinápticas entre neuronas de actividad similar. Los modelos conexionistas del aprendizaje han sido aplicados con éxito en al ámbito educativo (Adams, 1990; Seidenberg y McClelland, 1989).

\section{CONCLUSIÓN}

Ante este panorama tan diverso, son muchos los investigadores que propugnan la necesidad de combinar e integrar los diferentes enfoques representacionales. Pero también existen autores que sostienen que todos los enfoques computacionales son limitados y no constituyen una buena aproximación a la naturaleza de la mente al no considerar aspectos fundamentales del pensamiento.

Someramente, estos aspectos son los siguientes: el paradigma simbólicocomputacional supone una desnaturalización de la psicología ya que su fundamentación es tecnológica, no naturalista; la toma de postura funcionalista del paradigma simbólico hace que se considere el cerebro como un sistema de cómputo, cuyas peculiaridades y características carecerían de interés ya que sólo son importantes los programas que el cerebro ejecuta; no se tienen suficientemente 
en cuenta ni las emociones ni la función de la conciencia en el pensamiento; se desatiende la aportación del cuerpo al pensamiento y la acción; al poner el énfasis en lo individual se desplaza a un segundo plano lo social, por lo que no se considera el papel que desempeña el entorno en el pensamiento (de Vega, 1998; Thagard, 2008).

A pesar de los puntos débiles y limitaciones del paradigma simbólico-computacional, desde esta perspectiva se ha contribuido de forma significativa a los logros conseguidos por la psicología cognitiva en el conocimiento de la actividad mental, de tal forma que en las últimas décadas se ha avanzado más en estas cuestiones que en toda la breve historia de la psicología. Además, sobre algunas de las objeciones señaladas se está trabajando eficazmente en la actualidad.

Respecto al conexionismo, desde una perspectiva neurológica, las redes artificiales se asemejan a la estructura cerebral en el sentido de que constan de elementos simples que se excitan e inhiben entre sí. Sin embargo, las redes neuronales reales son mucho más complejas que las redes artificiales. En las redes neuronales reales son miles de millones las células nerviosas con millones de millones de conexiones. También las neuronas reales son mucho más complejas que las unidades de las redes artificiales; en éstas últimas, simplemente se pasa la activación de unas a otras, pero en las neuronas reales actúan decenas de neurotransmisores que proporcionan conexiones químicas, por lo que se debe entender el cerebro en términos no sólo eléctricos sino también químicos. Las neuronas reales experimentan modificaciones en sus propiedades sinápticas y no sinápticas que tienen mucho más alcance que lo que ofrece una neurona artificial.

Además, en las representaciones locales, cada unidad tiene una interpretación conceptual o proposicional especificable, cosa que no ocurre con las neuronas del cerebro. Las neuronas reales, a diferencia de las unidades de redes neuronales artificiales, tienen conexiones excitatorias o inhibitorias con otras neuronas, pero nunca tienen conexiones de los dos tipos a la vez. Son muchas más las neuronas entre las que el cerebro distribuye sus representaciones que las que se encuentran en las redes artificiales sean locales o distribuidas.

Por otra parte, no se ha constatado ninguna relación entre el aprendizaje por retropropagación y los procesos cerebrales, es decir, que el aprendizaje por retropropagación no se corresponde con ningún proceso cerebral conocido. Aunque en las redes neuronales reales se observa la propagación hacia adelante típica de las redes de retropropagación, todavía no se conoce ningún mecanismo neurológico mediante el que las mismas vías que alimentan la activación hacia delante se usen para propagar la corrección del error hacia atrás. En este sentido O Reilly y Munakata (2000) han propuesto un algoritmo de naturaleza biológica que se aproxima bastante a la retropropagación. 
Por consiguiente, debemos tener en cuenta que la mayoría de los modelos conexionistas no son más que una aproximación al comportamiento de las neuronas reales. Aún así, la analogía entre el cerebro y la mente computacional ha sido muy productiva y en la actualidad se están desarrollando modelos de procesos computacionales que se asemejan más a los procesos cerebrales. Aunque todavía los modelos conexionistas distan bastante de emular a las redes neuronales ha quedado demostrada, en la simulación de diversos experimentos, su importancia psicológica.

El procesamiento en serie que el modelo simbólico-computacional clásico describía, presentaba importantes dificultades y limitaciones para enfrentarse a ciertas condiciones que son muy frecuentes en el medio adaptativo de los humanos; por ejemplo, en situaciones en que es necesario considerar a la vez varias restricciones simultáneas o en aquellas situaciones en que se precisa reconstruir o recordar informaciones a partir de partes de ellas que pueden no ser correctas o en las que la información está parcialmente degradada. Además esta mente serial y simbólica se aleja mucho de la mente real a la hora de captar con rapidez los significados globales de las situaciones y también es mucho menos flexible que la mente real si sus esquemas y conceptos son tan discretos y rígidos como lo son las representaciones que emplean los ordenadores digitales. Por lo tanto, desde una perspectiva conexionista, el funcionamiento secuencial que proponía el paradigma clásico parece poco realista como modelo de funcionamiento del sistema nervioso.

Al igual que el cerebro, los sistemas conexionistas realizan multitud de cómputos en paralelo efectuados por una gran masa de unidades de procesamiento que operan simultáneamente; se trata de un procesamiento continuo a través de la red, muy diferente a la ejecución secuencial en pasos discretos de programación característica del paradigma simbólico.

Aunque desde el conexionismo se ha tratado de de ofrecer alternativas a las deficiencias y limitaciones de los enfoques cognitivos anteriores, desde su mismo inicio también el conexionismo planteaba sus propios puntos débiles y sus limitaciones como modelo de lo mental que Fodor y Pylyshyn (1988), defensores de paradigma computacional-representacional clásico, se apresuraron a destacar. Estos y otros autores (Broadbent, 1985) acusan a los modelos conexionistas de limitarse a utilizar las ideas de la psicología cognitiva clásica y de la inteligencia artificial. Otros (Papert, 1988) consideran que las estructuras propuestas por estos modelos carecen de un análisis teórico serio que asegure que el modelo funcionará cuando se reproduzca a tamaño real.

Aún así, en sus cincuenta años de existencia, las ciencias cognitivas han experimentado avances significativos. Hoy se sabe cómo diseñar sistemas complejos capaces de hacer inferencias lógicas; se han creado modelos por medio de 
sistemas que utilizan reglas y conceptos, para varios aspectos del uso del lenguaje y la resolución de problemas; se ha avanzado mucho en el conocimiento del pensamiento analógico combinando experimentos psicológicos y modelización computacional; el estudio de las imágenes ha dejado de ser marginal para ocupar un importante lugar en las investigaciones de disciplinas como la psicología, las neurociencias y la computación; y los modelos conexionistas del aprendizaje y la aplicación del procesamiento en paralelo en la satisfacción de restricciones, permiten explicar muchos fenómenos psicológicos.

La lógica, las reglas, los conceptos, las analogías, las imágenes y las redes conexionistas tienen, como hemos visto, tanto ventajas como limitaciones y en muchos de los casos deben complementarse entre sí. Para Thagard (2008) una buena teoría unificada debería dar cumplida cuenta de esta complementariedad proponiendo estructuras caracterizadas por: "(1) la riqueza sensorial de las imágenes y las conexiones, (2) la capacidad organizativa de los conceptos, los análogos y las imágenes, y (3) el poder de expresión verbal de las reglas y las proposiciones de la lógica formal" (p.210).

De cualquier forma, desde las ciencias cognitivas no se ha logrado elaborar una teoría unificada que permita explicar todos los fenómenos psicológicos. Algunos investigadores en este campo mantienen que la mente es un sistema lógico o de reglas, otros que conceptual, otros que de analogías, o de imágenes, o de redes conexionistas. Tal diversidad es comprensible ya que en cualquier caso la mente es un sistema complejo en el que coexisten clases de pensamiento muy diversas. Es precisamente esta complejidad y diversidad de la mente lo que dificulta la formulación de una teoría unificada. Además, el carácter multidisciplinar de las ciencias cognitivas exige que para lograr progresos se complementen los esfuerzos de los investigadores en distintas disciplinas integrando los resultados de la investigación en los distintos campos lo que supone una dificultad añadida. Por consiguiente no existe un único enfoque que pueda considerarse como la base teórica de las ciencias cognitivas en su conjunto por lo que ningún modelo computacional actual tiene la capacidad representacional para abarcar todo el pensamiento humano.

\section{REFERENCIAS}

Adams, M. J. (1990). Beginning to read. Cambridge, MA: MIT Press.

Amsel, A. (1989). Behaviorism, neobehaviorism, and cognitivism in learning theory. Hillsdale, NJ: Erlbaum.

Anderson, J. R. (1983). The architecture of cognition. Cambridge, MA: Harvard University Press.

Anderson, J. R. (1993). Rules of the mind. Hillsdale, NJ: Erlbaum.

Anderson, J. R., Qin, Y., Stenger, V. A., y Carter, C. S. (2004). The relationship of three cortical regions to an information-processing model. Cognitive Neu- 
roscience, 16, 637-653.

Ashby, F. G. y Waldron, E. M. (2000). The neuropsychological bases of category learning. Current Directions in Psychological Science, 9(1), 10-14.

Ballesteros, S. (1992). La representación del conocimiento en los sistemas conexionistas. Psicothema, 4(2), 343-354.

Barsalou, L. W. (1983). Ad hoc categories. Memory and Cognition, 11(3), 211227.

Barsalou, L. W., Simmons, W. K., Barbey, A. K. y Wilson, C. D. (2003). Grounding conceptual Knowledge in modality-specific systems. Trends in Cognitive Sciences, 7(2), 84-91.

Bates, E. A. y Elman, J. L. (2002). Connectionism and the study of change. En M. H. Johnson, Y., Munakata y R. O. Gilmore (eds.), Brain development and cognition (pp.420-440). Oxford: Blackwell.

Boroojerdi, B., Phipps, M., Kopylev, L., Wharton, C. M., Cohen, L. G. y Grafman, J. (2001). Enhancing analogic reasoning with RTMS over the left prefrontal cortex. Neurology, 56, 526-528.

Broadbent, D. E. (1985). A question of levels: Comments on McClelland and Rumelhart. Journal of Experimental Pschology: General, 114, 189-192.

Chomsky, N. (1972/1986). El lenguaje y el entendimiento. Barcelona: Seix Barral.

Chomsky, N. (1988). Lenguage and problems of knowledge: The Managua lectures. Cambridge, MA: MIT Press.

Chomsky, N. (2002/2003). Sobre la naturaleza del lenguaje. Madrid: Cambridge University Press.

Christoff, K., Pabhakaran, V., Dorfman, J., Zhao, Z., Kroger, J. K., Holyoak, K. J. y Gabrieli, J. D. K. (2001). Rostrolateral prefrontal cortex involvement in relational integration during reasoning. NeuroImage, 14(5), 1136-1149.

Churchland, P. S. y Sejnowski, T. (1992). The computational brain. Cambridge, MA: MIT Press.

Clark, A. (1998). Embodied, situated, and distributed cognition. En W. Bechtel and G. Graham (eds.), A companion to cognitive science (pp. 506-517). Malden, MA: Blackwell.

Collins, A. M. y Loftus, E. F. (1975). A spreading-activation theory of semantic processing. Psychological Review, 82, 407-428.

Cummins, R (1983). The nature of psychological explanation. Cambridge, Mass.: MIT Press.

Dehaene, S., Spelke, E., Pinel, P., Stanescu, R. y Tsivkin, S. (1999). Sources of mathematical thinking: Behavioral and brain-imaging evidence. Science, 284(5416), 970-974.

Eliasmith, C. y Thagard, P. (2001). Integrating structure and meaning: A distributed model of analogical mapping. Cognitive Science, 25(2), 245-286.

Fodor, J. A. (1985). El lenguaje del pensamiento. Madrid: Alianza.

Fodor, J. A. (1988). Psychosemantics. The Problem of meaning in the Philosophy of Mind. Cambridge, Mass.: MIT Press. 
Fodor, J. A. y Pylyshyn, Z. (1988). Connectionism and cognitive architecture: A critical análisis. Cognition, 28, 3-71.

Funt, B. (1980). Problem solving with diagrammatic representations. Artificial Intelligence, 13(3), 201-230.

Holland, J. H., Holyoak, K. J., Nisbett, R. E. y Thagard, P. R. (1986). Induction: Processes of inference, learning, and discovery. Cambridge, MA: MIT Press.

Holyoak, K. J. y Thagard, P. R. (1995). Mental leaps: Analogy in creative thought. Cambridge, MA: MIT Press/Bradford Books.

Hummel, J. E. y Holyoak, K. J. (1997). Distributed representations of structure: A theory of analogical acces and mapping. Psychological Review, 104(3), 427-466.

Hummel, J. E. y Holyoak, K. J. (2003). A symbolic-connectionist theory of relational inference and generalization. Psychological Review, 110(2), 220-264.

Johnson, M. y Lakoff, G. (2002). Why cognitive linguistics requires embodied realism. Cognitive Linguistics, 13(3), 245-263.

Keil, F. (1989). Concepts, kinds, and cognitive development. Cambridge, MA: MIT Press/Bradford Books.

Kintsch, W. (1988). The role of knowledge in discourse comprension: A construction-integration model. Psychological Review, 95(2), 163-182.

Kintsch, W. (1998). Comprehensión: A paradigm for cognition. Cambridge: Cambridge University Press.

Kolodner, J. (1993). Case-based reasoning. San Mateo, CA: Morgan Kaufmann. Kosslyn, S. M. (1980). Image and mind. Cambridge, MA: Harvard University Press.

Kosslyn, S. M. (1994). Image and brain: The resolution of the imagery debate. Cambridge, MA: MIT Press.

Kosslyn, S. M. y Shwartz, S. P. (1977). A simulation of visual imagery. Cognitive Science, 1, 265-295.

Kosslyn, S. M. y Koenig, O (1992). Wet mind: The new cognitive neuroscience. Nueva York: Free Press.

Kosslyn, S. M., Ganis, G. y Thompson, W. L. (2001). Neural foundations of imagery. Nature Reviews Neuroscience, 2, 635-642.

Kroger, J. K., Sabb, F. W., Fales, C. L., Bookheimer, S. Y., Cohen, M. S. y Holyoak, K. J. (2002). Recruitment in anterior dorsolateral prefrontal cortex in human reasoning: A parametric study of relational complexity. Cerebral Cortex, 12(5), 477-485.

Kunda, Z. y Thagard, P. (1996). Forming impressions from stereotypes, traits, and behaviors: A parallel-constrained-satisfaction theory. Psychological Review, 103(2), 284-308.

Lakoff, G. (1987). Women, fire, and dangerous things. Chicago: Chicago University Press.

Lakoff, G. (1994). What is metaphor? En J. A. Barnden y K. J. Holyoak (eds.), Advances in connectionist and neural computation theory. Vol. 3, Analogy, metaphor, and reminding (pp. 203-257). Norwood, NJ: Ablex. 
Lakoff, G. y Johnson, M. (1986). Metáforas de la vida cotidiana. Madrid: Cátedra.

Langacker, R. W. (1987). Foundations of cognitive grammar. Stanford, CA: Stanford University Press.

Larkin, J. H. y Simon, H. A. (1987). Why a diagram is (sometimes) worth ten thousand words. Cognitive Science, 11(1), 65-100.

Leake, D. B. (ed.) (1996). Case-based reasoning: Experiences. Lessons, and future directions. Menlo Park, CA: AAAI Press/MIT Press.

Marslen-Wilson, W. (1989). Access and integration: Projecting sound onto meaning. En W. Marslen-Wilson (ed.), Lexical representation and process (pp. 3-24). Cambridge, MA: MIT Press.

McCawley, J. D. (1993). Everything that linguist have always wanted to know about logic-but were ashamed to ask. Chicago: University of Chicago Press.

McClelland, J. L. y Rumelhart, D. E. (1981). An interactive activation model of context effects in letter perception: Part. 1. An account of basic findings. Psychological Review, 88(5), 375-407.

McClelland, J. L. y Elman, J. L. (1986). The TRACE model of speech perception. Cognitive Psychology, 18(1), 1-86.

McClelland, J. L. y Patterson, K. (2002). Rules of connections in past-tense inflections: What does the evidence rule out? Trends in Cognitive Sciences, 6(11), 465-472.

McWhinney, B. y Leinbach, J. (1991). Implementations are not conceptualizations: Revising the verb model. Cognition, 40(1), 121-157.

Medina, N. (2008). La ciencia cognitiva y el estudio de la mente. Revista de Investigación en Psicología, 11(1), 183-198.

Miller, G. A. (1991). The science of words. Nueva York: Scientific American Library.

Minsky, M. (1975). A framework for representing knowledge. En P. H. Winston (ed.), The psychology of computer vision (pp. 211-277). Nueva York: McGraw-Hill.

Murphy, G. L. (2002). The big book of concepts. Cambridge, MA: MIT Press.

Murphy, G. L. y Medin, D. (1985). The role of theories in conceptual coherence. Psychological Review, 92(3), 289-316.

Newell, A. (1973). Production systems: Models of control structures. En W. G. Chase (ed.), Visual information processing (pp. 463-526). New York: Academic Press.

Newell, A. (1990). Unified theories of cognition. Cambridge, MA: Harvard University Press.

Newell, A., Shaw, J. C. y Simon, H. A. (1958). Elements of a theory of human problem solving. Psychological Review, 65(3), 151-166.

Newell, A. y Simon, H. A. (1972), Human problem solving. Englewood Cliffs, NJ: Prentice-Hall.

O'Reilly, R. C. y Munakata, Y. (2000). Computacional explorations in cognitive neuroscience: Understanding the mind by simulating the brain. Cambridge, 
MA: MIT Press.

Paivio, A. (1971). Imagery and verbal processes. Nueva York: Holt, Rinehart, and Winston.

Paivio, A. (1986). Mental representations. New York: Oxford University Press.

Palmer, S. E. y Kimchi, R. (1986). The information processing approach to cognition. En T. J. Knapp y L. C. Robertson (eds.), Approaches to cognition: Contrasts and controversies (pp. 37-77). Hillsdale, NJ: Erlbaum.

Papert, S. (1988). One AI or many? Daedalus, 117, 1-14.

Pinker, S. (2007). Cómo funciona la mente. Barcelona: Destino.

Pinker, S. y Prince, A. (1988). On language and connectionism: Analysis of a parallel distributed processing model of language acquisition. Cognition, 28(1-2), 73-193.

Pinker, S. y Ullman, M. T. (2002). The past and future of the past tense. Trends in Cognitive Sciences, 6(11), 456-464.

Pylyshyn, Z. (1984). Computation and cognition: Toward a foundation for cognitive science. Cambridge, MA: MIT Press.

Pylyshyn, Z. (2002). Mental imagery: In search of a theory. Behavioral and Brain Sciences, 25(2), 157-237.

Rosenbloom, P. S., Laird, J. E. y Newell, A. (eds.) (1993). The Soar papers: Research on integrated intelligence. Cambridge, MA: MIT Press.

Rumelhart, D. E. (1989). The architecture of mind: A connectionist approach. En M. I. Posner (ed.), The architecture of cognition (pp. 133-156). Cambridge, MA: MIT Press.

Rumelhart, D., McClelland, J. L. and PDP Research Group (1986). Parallel distributed processing: Explorations in the microstructure of cognition, Vol.1: Foundation. Cambridge, Mass.:MIT Press.

Schacter, D. L., Cooper, L. A. y Delaney, S. M. (1990). Implicit memory for unfamiliar objects depends on access to structural descriptions. Journal of Experimental Psychology: General, 119, 5-24.

Seidenberg, M. S. y McClelland, J. L. (1989). A distributed, developmental model of word recognition and naming. Psychological Review, 96(4), 523-568.

Shepard, R. N. y Metzler, J. (1971). Mental rotation of three-dimensional objects. Science, 171, 701-703.

Shepard, R. N. y Cooper, L. A. (1982). Mental images and their transformations. Cambridge: MIT Press.

Smolensky, P. (1988). On the proper treatment of connectionism. Behavioral and Brain Sciences, 11, 1-74.

Smythe, W. E. (1988). The semantic distinction between propositional and skillful knowing. Journal of Intelligent Systems, 1(2), 135-184).

Smythe, W. E. (1989). The case for cognitive conservatism: A critique of Dan Lloyd's approach to mental representation. Behaviorism, 17(1), 63-73.

Spellman, B. A. y Holyoak, K. J. (1993). An inhibitory mechanism for goaldirected analogical mapping. En Proceedings of the Fifteenth Annual Conference of the Cognitive Science Society (pp.947-952), Hillsdale, NJ: Erlbaum. 
St. John, M. F. (1992). The story gestalt: A model of knowledge-intensive processes in text comprehension. Cognitive Science, 16(2), 271-306.

Stabler, E. P. (1992). The logical approach to syntax. Cambridge, MA: MIT Press.

Thagard, P. (2008). La mente. Introducción a las ciencias cognitivas. : Buenos Aires: Katz Editores.

Touretzky, D. y Hinton, G. (1988). A distributed connectionist production system. Cognitive Science, 12(3), 423-466.

Vega de, M. (1998). La psicología cognitiva: ensayo sobre un paradigma en transformación. Anuario de Psicología, 29(2), 21-44.

Wharton, C. M., Holyoak, K. J., Downing, P. E., Lange, T. E., Wickens, T. D. y Melz, E. R. (1994). Below the surface: Analogical similarity and retrieval competition in reminding. Cognitive Psychology, 26(1), 64-101. 